\section{Patterns in Drying Water Films}

\author{
Stephen G. Lipson, Nirit Samid-Merzel and David S. Tannhauser \\ Israel Institute of Technology
}

\begin{abstract}
Why do apparently uniform and simple systems spontaneously develop complex patterns? The study of drying water films is helping us to understand how a departure from equilibrium affects the type of pattern formed
\end{abstract}

Some patterns appear under equilibrium conditions, such as domains in a ferromagnet, and can be explained in terms of the physical properties of the medium in their move to achieve minimal total free energy. Other well-known patterns only arise when the system moves out of equilibrium, such as the polygonal pattern of cracks in a drying mud flat or the Giant's Causeway (see page 116), or the periodic wind-blown waves on the desert sand. The most famous example is the snowflake, which has an intricate shape determined in some complicated way during its growth, and which bears little relation, apart from the six-fold symmetry, to the equilibrium crystal - a hexagonal prism. Typical features of non-equilibrium patterns can be described and often understood in general terms, but pinning them down quantitatively has turned out to be much harder.

Dynamic patterns arise in general when the equations of motion of a medium have runaway solutions for certain ranges in space and time, so that these solutions become dominant at long times even when the system is initially perturbed by only infinitesimal fluctuations. Over 100 years ago Lord Rayleigh explained the breakup of a water jet into drops by calculating the rate of growth of surface undulations on the cylindrical jet, and choosing the fluctuation which grew most quickly. This method, called 'linear stability analysis' is still used as a first approximation to understanding patterns. In recent years many apparently simple and homogeneous media, such as uniform viscous fluids, reacting chemicals and bacterial colonies, have been found to develop surprisingly intricate patterns when their equilibrium is disturbed. Their study from both experimental and theoretical standpoints has been prolific, especially since the availability of fast computers has made detailed simulations possible and thus the 'solution' of very complicated differ- ential equations. In particular, it is found that patterns produced in widely differing media often seem to have similar features, and one would like to understand the physics underlying this apparent unification. (One could even claim that life itself is a dynamic instability which has developed in the earth's physicochemical environment.)

One common problem that inevitably arises is finding systems which are sufficiently simple so that both experimental data and theoretical analyses of them can be compared. A thin film of water evaporating from a mica substrate (clean and atomically flat) is one such example. Surprisingly, it separates into two phases like a freezing liquid, and the separation is slow and controllable. Moreover, the system is two-dimensional and isotropic.

A dynamical process which is of great practical importance is phase separation, for example, solidification from a melt. The thermodynamics of the solid-liquid equilibrium are well understood, but the process by which that equilibrium is achieved are still under discussion. A solid crystal can only grow from the melt under a certain degree of supercooling; first a microscopic germ has to nucleate, and then the degree of supercooling controls the rate at which it develops. Early in its growth, a crystal nucleated far from the boundaries of the fluid container will usually develop interface instabilities. Then the type of pattern obtained depends greatly on the degree of supercooling and the physical properties of the solid crystal and the liquid-solid interface. Crystals are anisotropic and even the smallest directional variations in their properties can affect runaway solutions crucially. As a result, the problem of determining growth patterns in solidification, which generally leads to dendritic (branched, or tree-like) growth, has been very involved. In get-

\section{Phase-separation}

ting realistic solutions, numerical computations have been heavily used, but because of the need to specify anisotropic parameters it has been difficult to see the wood for the trees. In attempts to understand the basic physics behind solidification patterns, it would be nice to be able to study completely isotropic materials. Growth simulations for the isotropic case have been carried out in two dimensions by Ofer Shochet, Raz Kupferman and Eshel Ben-Jacob at TelAviv University and by Thomas Ihle and Heiner Müller-Krumbhaar at Jülich, who independently pointed out a 'fingerprint' characteristic of isotropic conditions, which they called 'parity-broken dendrites' or 'doublons' because they consist of two asymmetric parts related by mirror symmetry. Although hints of their existence could be found in some experiments on crystal growth, the fingerprint was generally buried under the effects of anisotropy. This is where the water films come in.

\section{Water films}

In the course of experiments on ice nucleation, done together with Michael Elbaum, we discovered a new phase-separation phenomenon in thin pure water films on a mica substrate. The development of these films, which appear to be quite isotropic in the substrate plane, has proved an almost ideal environment to study pattern formation during phase separation under a wide variety of circumstances. We have so far used simple optical interference microscopy for these studies, although it has become clear that a more delicate tool such as ellipsometry is really necessary.

What are the two phases involved? Water films deposited on an atomically clean (cleaved) mica surface are adsorbed by two mechanisms. The first is the well-known van der Waals interaction which exists between any two materials, and the second is a short-range dipole interaction more specific to water and ionic crystals. When these two forces have opposite signs, an interesting situation occurs. In the strict sense of the term, water 'wets' mica, ie a uniform thick liquid layer equilibrates with saturated vapour. But when the water layer becomes thin enough (about 25 nanometres) by evaporation, dewetting can occur, leaving behind a molecularly thin layer (maybe 2 nanometres) of water on the mica. One can envisage here a potential application for ellipsometry, an opti- 

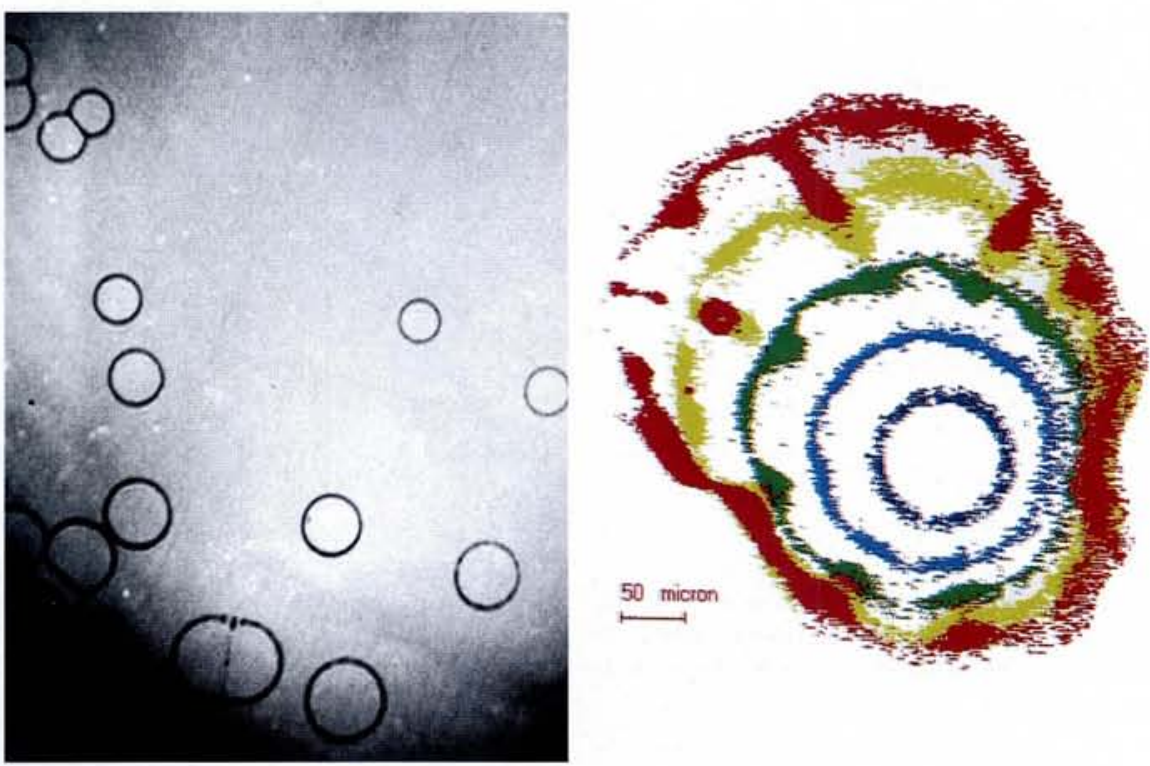

Fig 1 left Several dry patches nucleate in an evaporating water film about 25 nanometres thick. Water from each patch is expelled into its rim, which can be seen as a dark ring. Right we show the evolution of a rim and its instabilities at 40 second intervals, each image being coloured differently. Initially the rim is circular but eventually it develops into the star-like patterns of fig 2

cal technique which is able to measure the thickness and properties of films with nanometer accuracy.

The dewetting scenario can be cast into the form of a phase-separation, in which the two phases are the 'thick' and 'thin' films, which are analogous to 'liquid' and 'solid' respectively, since the thick film is fluid and the thin one is not. The pressure of the water vapour drives the phase transition; reducing the pressure causes 'freezing' in this case. Let us look first at the equilibrium properties $($ box 1$)$. At relatively high vapour pressure, close to saturation, the vapour can equilibrate with a thick film, and at very low pressure with a very thin film. At intermediate pressures, the vapour can coexist with both films, but one film is usually metastable; only at one pressure is there equilibrium between the vapour and both films, and this is equivalent to the triple point in the liquid-gas-solid system.

Nucleation in the solidification model is then equivalent to nucleation of a 'thin' patch which, because of the isotropy of the medium, has circular shape to minimize its edge energy. As it grows its perimeter becomes unstable, for the same reasons as Rayleigh's water jet, and the characteristic shapes arise (figure 1).

Experimentally, the water films are grown on cleaved mica in a vacuum chamber containing water vapour. The vapour pressure is controlled by main- taining a bath of water or ice at a fixed temperature $T_{1}$. The mica surface is a temperature $T$ somewhat lower than $T_{1}$ so that water condenses on it, whereas the surrounding chamber is kept somewhat warmer than $T_{1}$ so that it stays dry. The mica is observed through a window in the cell by a microscope with quasimonochromatic illumination using interference contrast, which is effective since the refractive index of water is close to the geometric mean of those of mica and the vapour. Dark fringes occur when the water layer has thickness equal to an odd number of quarter waves, 110 nanometres in our case. The interference can be
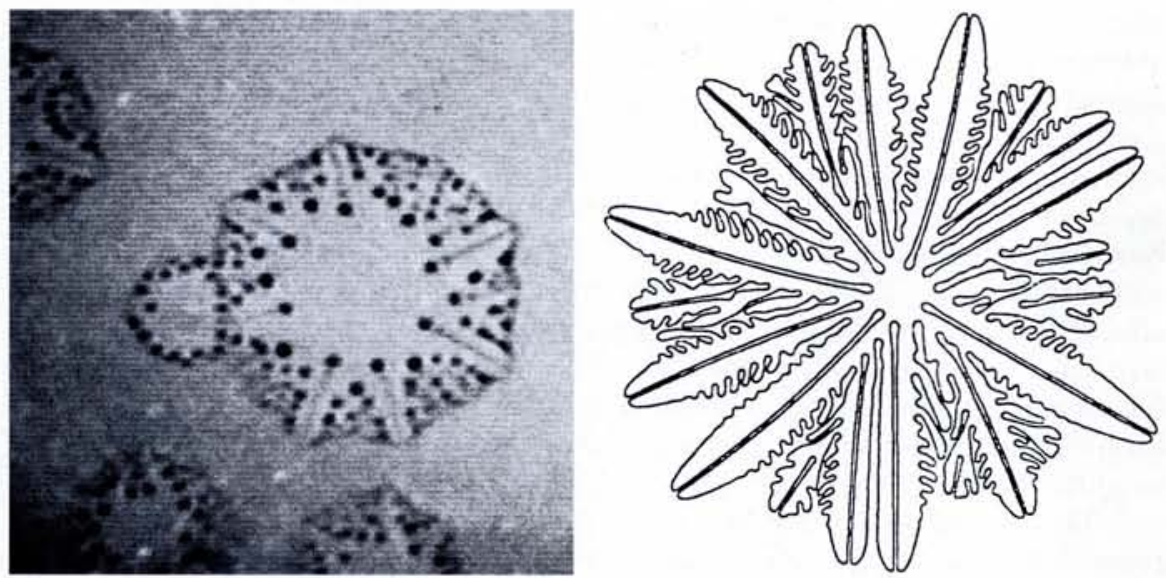

Fig 2 Growth of the instability around a dry patch leads to a star-like pattern, several of which can be seen at different stages of growth in the photograph. The adjacent simulation by Ben-Jacob's group shows the interface between a liquid and an isotropic solid grown at high supercooling, and is surprisingly similar.The star-like structure can be seen to be built up from rows of drops radiating from the centre of the patch. These are caused by a type of Rayleigh instability, which would only occur in the simulation at a much later time 


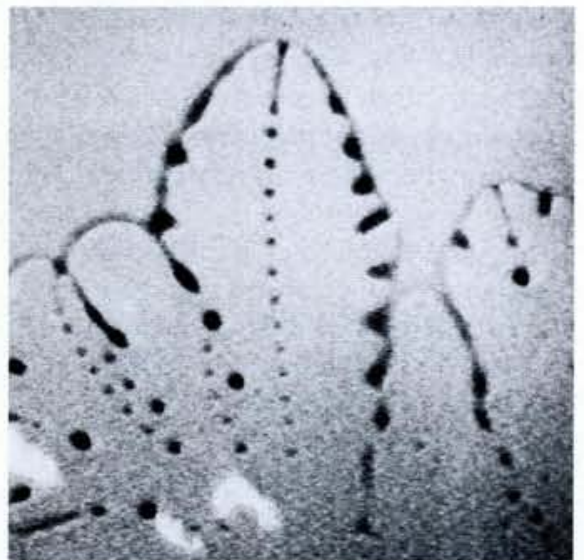

Fig 3 Observing the details of an expanding rim shows features which have been called 'doublons' or 'parity-broken dendrites' and are characteristic of growth of an isotropic solid from the melt. They can be seen in the simulation in fig 2, and also feature in those by Müller-Krumbhaar's group shown here. At the top left of the outermost profile in fig 1 right one of these features can be seen developing

patterns of drops evolve. Similar patterns of drops have also been observed by Günter Reiter at Mulhouse, France and others in the rupture of polymer films.

\section{Behaviour of evolving films}

In order to see the relationship between solidification models and film evolution, both in two dimensions, one must write down the relevant equations (box 2).

These not only explain why the patterns are similar, but also indicate regions in which they are expected to differ.

The basic physics of the solidification process is very simple, even if solution of the problem is difficult. When the solid grows from a supercooled fluid, latent heat is emitted, some of which goes into heating the solid up to the melting point, and the rest is redistributed into the surroundings by heat diffusion in the isotropic fluid. At the crystal surface, the growth velocity is controlled by the rate at which latent heat flows down the local normal temperature gradient. Anisotropy comes into the process through effects such as surface tension; the local melting point is depressed by an amount proportional to the product of surface tension and local curvature (the Gibbs-Thomson effect). Although the variation of the surface tension may be only a few percent between surface orientations where it is maximum and minimum, this can be magnified by the runaway solutions.

In the water films, although the environment may seem to be very different, we can establish an analogous situation. A thin patch nucleates only when the film is supercooled, ie thinner than equilibrium thickness. Some of the water which was in the patch evaporates, but most of it is ejected (like the latent heat) into the surrounding region where it is first used to build up the rim to equilibrium thickness or higher, and the excess is redistributed into the film. Growth of the patch is maintained as long as the film continues to evaporate. It is the manner by which the water redistributes which is problematic, because the process is surely governed by hydrodynamics, and not by diffusion. But under evaporating conditions, when one takes into account the fact that the vapour pressure over a curved surface is modified by the Gibbs-Thomson effect, it is easy to show that a diffusion-like term is added to the hydrodynamics, and for thin enough films can be shown to be dominant. In this way we establish an almost complete analogy between the film evolution and solidification, when the film is thin enough. This is why the evolution in the two situations looks so similar, as we saw in figures 2 and 3 .

Notice that there is no in-plane anisotropy in this case; both phases are liquid, and the substrate crystallinity seems to have no influence. By varying the evaporation rate we can easily get into two other regimes. At very low rates (close to equilibrium) the hydrodynamic redistribution dominates, giving an analogy to viscous fingering patterns, which appear when an inviscid fluid is forced under pressure into a viscous one (figure 4). At very high rates the system quenches into an unstable region, where phaseseparation is spontaneous and no nucleation of patches is necessary. This is analogous to spinodal decomposition of an alloy and patterns of drops develop (figure 5) which have similar geometry to

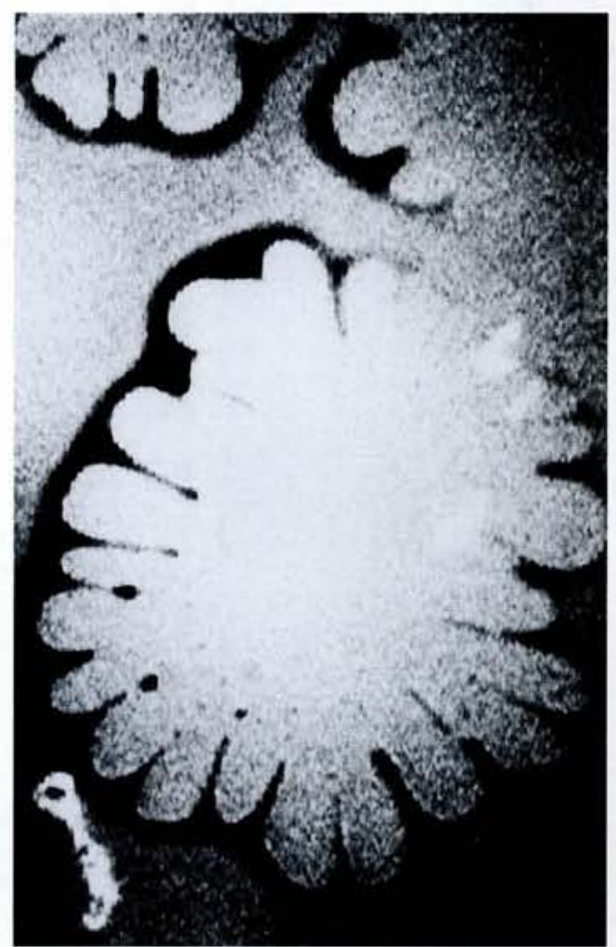

Fig 4 above At low evaporation rates the rim of a dry patch left develops a form typical of viscous fingering patterns, produced for example when air is forced into a layer of viscous fluid constrained between two parallel plates above right. This is an effect determined by surface tension and hydrodynamics Photograph courtesy of E. Ben-Jacob

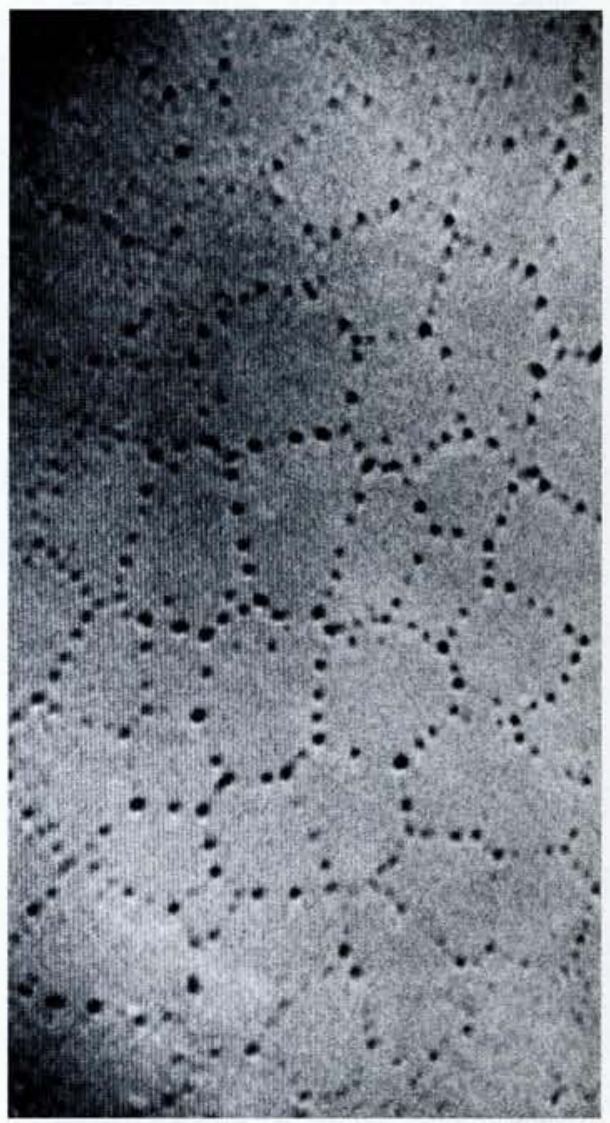




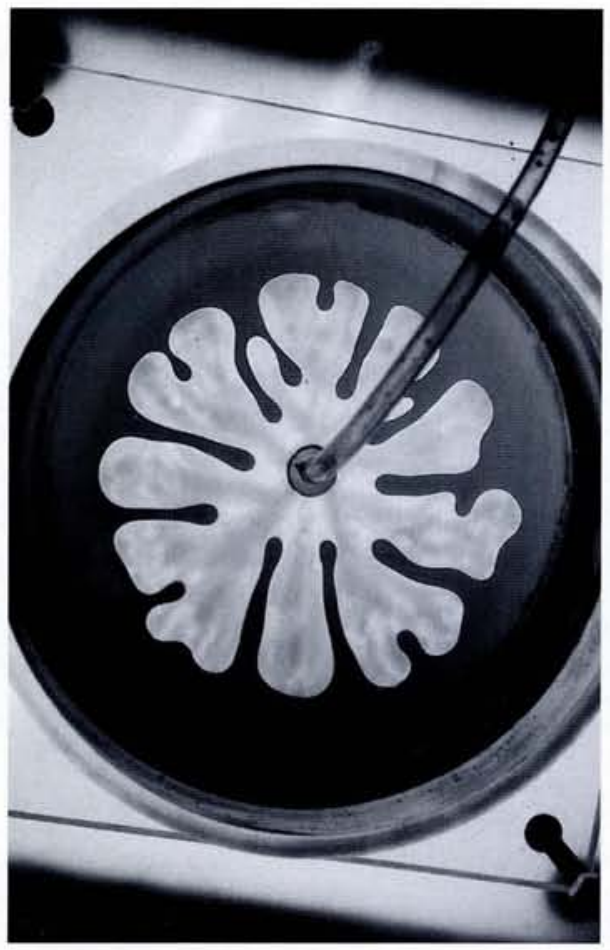

Fig 5 below At the other limit, of very high evaporation rates, many dry patches are created without a nucleation barrier; this is equivalent to spinodal decomposition in an alloy. The final result is a drop pattern outlining a polygonal structure left very similar to the cracks in drying mud below

Photograph courtesy of H. Lipson

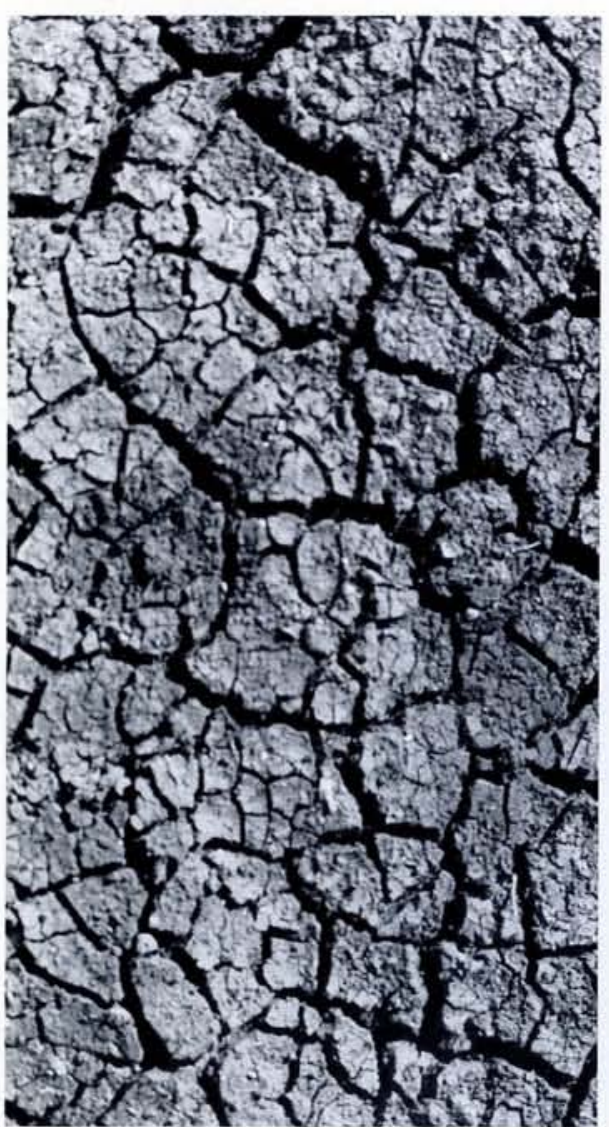

Box 1

Analytically, we describe the interaction between the film (thickness $h$ ) and the substrate by a free energy $g_{i}(h)$ per unit area. The van der Waals term is proportional to $h^{-2}$ and the polar interaction term to $\exp \left(-h / d_{\mathrm{o}}\right)$ so we can write

$g_{i}(h)=A h^{-2}-B \exp \left(-h / d_{0}\right)$

This model has been studied by Ashutosh Sharma at Kanpur, Indai and here we assume both $A$ and $B$ to be positive. Now the chemical potential of the fluid is $\mu_{\text {fluid }}=\rho^{-1} \mathrm{~d} g_{i} / \mathrm{d} h$ since adding unit volume to the film adds $\rho$ molecules. In equilibrium $\mu_{\text {fluid }}=\mu_{\text {vapour }}=k_{B} \ln \left(p / p_{\text {sat }}\right)$, from which one immediately sees that

$\mathrm{d} g_{i} / \mathrm{d} h=\rho \mu_{\text {vapour }}$

ie $\Psi \equiv g_{i}-h \rho \mu_{\text {vapour }}$ is minimized. Now, looking at $\Psi(h)$ for various values of the external constraint $\mu_{\text {vapour }}$ (figure 6) we see that there are in general two minima, representing two phases with different film thicknesses. As $\mu_{\text {vapour }}$ is varied a whole range of possibilities appears, from which the phase diagram can be deduced. In particular, at one vapour pressure the two minima are at the same level and there is equilibrium between the two phases and the vapour, whereas otherwise one phase is metastable. The hump between the two minima represents a nucleation barrier for the formation of a new phase. In the region where $\mathrm{d}^{2} g_{i} / \mathrm{d} h^{2}$ is negative, spinodal (non-nucleated) decomposition occurs, as in binary alloys

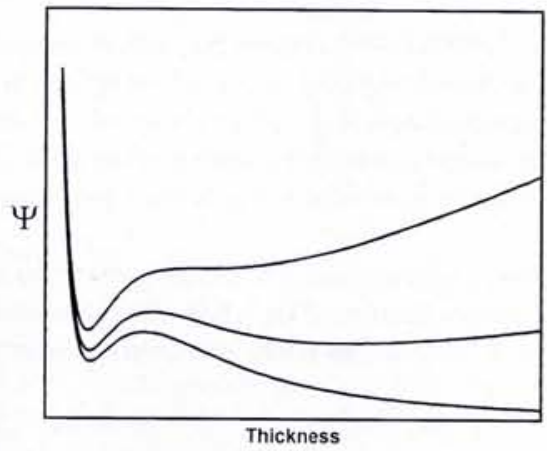

Fig 6 The interface energy $g_{i}(h)$ and the thermodynamic potential $\Psi=g_{i}-h \rho \mu_{\text {vapour }}$ for the model interaction. For any $\mu_{\text {vapour }}<0, \Psi(h)$ has at least one minimum which represents an equilibrium state of the film at that thickness. The lowest curve represents saturated vapour pressure, where $\Psi=g_{i}$ and has minimum value at infinite thickness: wetting. For a range of $\mu_{\text {vapour }}$ however, illustrated by the middle curve, there are two minima showing that two states can coexist, although one is usually metastable. Nucleation of the stable state corresponds to thermal excitation across the barrier separating it from the metastable one. When the barrier becomes zero (upper curve) a non-nucleated (spinodal) transition occurs

Box 2

The dynamics of the film can be described by an evolution equation for $\mathrm{d} h / \mathrm{d} t$. For small $|\nabla h|$, the curvature is approximated by $\nabla^{2} h$ and hydrodynamic flow is driven by gradients in chemical potential. There are two sources for this: one is $\rho^{-1} \mathrm{~d} g_{i} / \mathrm{d} h$, and the other is the Gibbs-Thomson pressure $\gamma \nabla^{2} h$. Assuming no slip at the interface with the substrate, the response to a gradient is given by

$\mathrm{d} h / \mathrm{d} t=\nabla \cdot\left[\left(h^{3} / 3 \eta\right) \nabla \rho \mu\right]$

Evaporation gives an additional term $\mathrm{d} h / \mathrm{d} t=-\alpha\left(\mu-\mu_{\text {vapour }}\right)$ in which $\alpha$ is a constant which is related to kinetic theory of gases. Together, these lead to the differential equation

$$
\mathrm{d} h / \mathrm{d} t=\nabla \cdot\left\{\left(h^{3} / 3 \eta\right) \nabla\left[\left(\mathrm{d} g_{i} / \mathrm{d} h\right)-\gamma \nabla^{2} h\right]-(\alpha / \rho)\left[\left(\mathrm{d} g_{i} / \mathrm{d} h\right)-\gamma \nabla^{2} h-\rho \mu_{\text {vapour }}\right]\right.
$$

The term $\left(\mathrm{d} g_{i} / \mathrm{d} h\right)-\rho \mu_{\text {vapour }}$ is exactly zero at equilibrium, and its value is often negligible on the time scale of an experiment. One can then see, without going into too much detail, that in the limit of thin films, $\mathrm{h} \rightarrow 0$, the remaining terms represent a diffusion equation (with diffusion constant $\alpha \gamma / \rho$ ), whereas at large $h$ the dominant terms are those for a hydrodynamic flow situation. The water film can therefore be usefully compared to other experiments in either limit 
the patterns of cracks in drying mud.

Another prominent effect which can easily be investigated with the water film system during the later stages of film evolution is the dynamics of the dropforming instability. Down the symmetry axis of a doublon there is a strip of water which is unstable to drop formation, in much the same way as Rayleigh's varicose instability of the cylindrical water jet. But because the shape is so different (it is like a carrot peeling, because the effective contact angle is only about $0.5^{\circ}$ ) the instability develops slowly, during tens of seconds, and is ideal for detailed study.

Optical interferometry is being used in our laboratory for studying a variety of pattern-formation processes. A major use, developed during the past several years, has been to investigate in detail the fields which drive crystal growth.
When a crystal grows from aqueous solution, the refractive index of the solution varies (approximately linearly) with the concentration, and by observing crystals growing in a thin cell under an interference microscope we have been able to visualize and measure quantitatively the concentration fields around the crystal. Results of these experiments are being used to check theoretical work on the dendritic instability. In other experiments, optical tomography is being used to measure temperature fields in three dimensions around ice crystals growing from the melt and polarization microscopy to visualize patterns of magnetic flux in type-I superconductors. Together with colleagues working on theoretical aspects of these problems, we hope to understand more about how these intricate and fascinating structures evolve.
This research was supported by the German Israel Foundation and the Minerva Centre for Non-linear Science

\section{Further reading}

J. Israelachvili Intermolecular and Surface Forces and edition (Academic Press, London, 1991)

Eshel Ben-Jacob From Snowflake Formation to Growth of Bacterial Colonies, Contemporary Physics 34 (1993) $247-73$

N. Samid-Merzel, S.G. Lipson and D.S. Tannhauser Phys. Rev. E 57 (1998) 2906-13

T. Ihle and H. Müller-Krumbhaar Phys. Rev. Lett. 70 (1993) 3083

Ashutosh Sharma Langmuir 9 (1993) 861

\section{The Giant's Causeway - a Polygonal Puzzle}

Among the many cellular patterns that occur in nature, few have been the subject of as much debate as the Giant's Causeway. This formation of tightly packed polygonal basalt columns is exposed by the action of the sea in a particularly scenic setting in Northern Ireland, but the phenomenon is widespread elsewhere as well. For several centuries savants have asked whether this beautiful pattern is due to crystallization, compaction, convection,or simply cracking of the original lava flow as it cooled and contracted. If these are just cracks, why should they take such an elegant form, without the T-junctions that we see in mud cracks, wherever a new crack meets an old one?

The answer seems to lie in the very slow vertical propagation of the crack patterns as the thick flow cools over a long period of time. What we see is the asymptotic state of the crack pattern, after it has propagated through perhaps a hundred metres of basalt. Like the atoms of a glass (see Europhysics News 292 2) the cracks settle into an arrangement which is locally quite well organised, but random over longer distances.

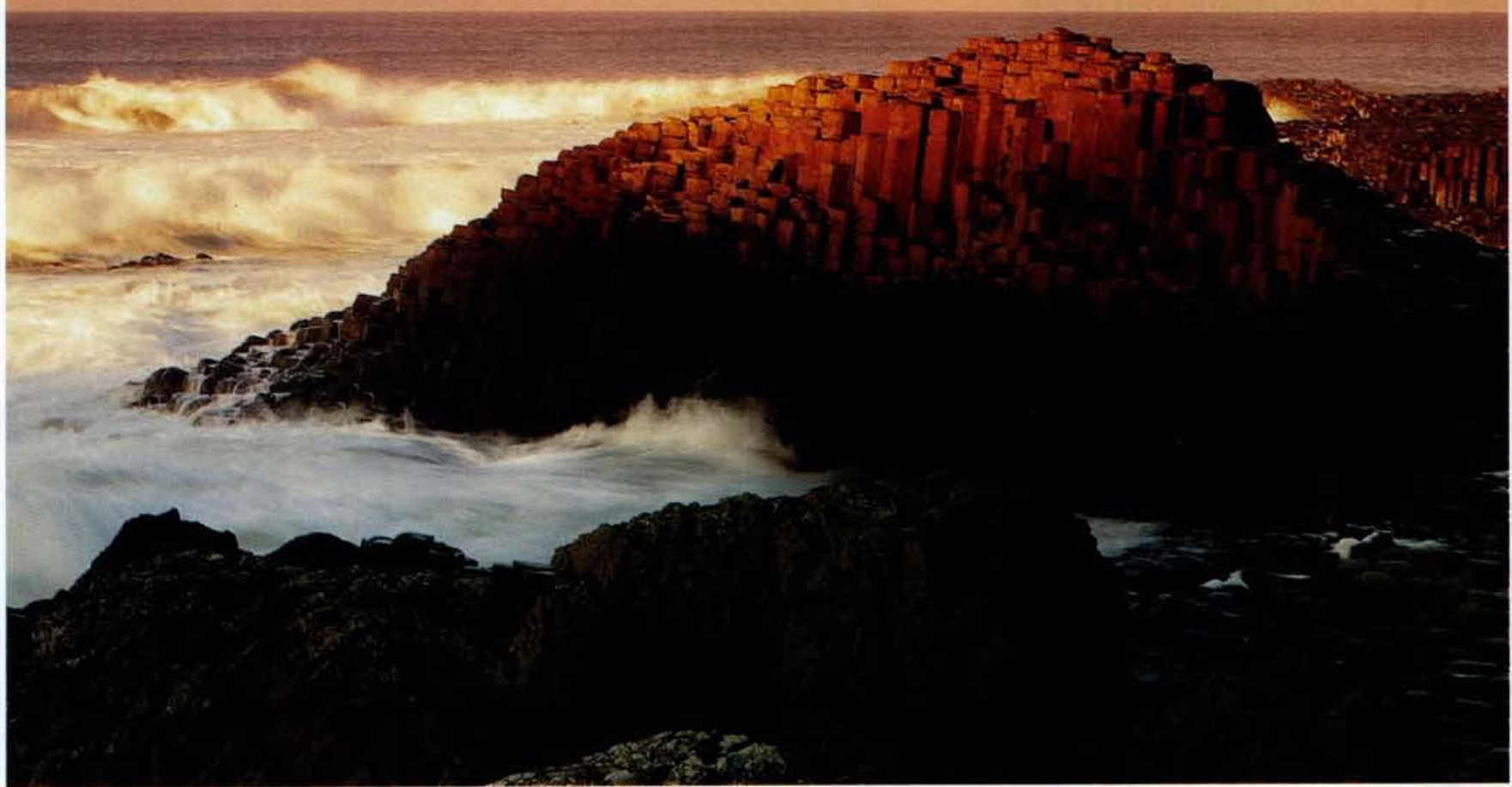

